\title{
SAKK 16/14: Durvalumab in Addition to Neoadjuvant Chemotherapy in Patients With Stage IIIA(N2) Non-Small-Cell Lung Cancer-A Multicenter Single-Arm Phase II Trial
}

Rothschild, Sacha I ; Zippelius, Alfred ; Eboulet, Eric I ; Savic Prince, Spasenija ; Betticher, Daniel ; Bettini, Adrienne ; Früh, Martin ; Joerger, Markus ; Lardinois, Didier ; Gelpke, Hans ; Mauti, Laetitia

A ; Britschgi, Christian ; Weder, Walter ; Peters, Solange ; Mark, Michael ; Cathomas, Richard ;

Ochsenbein, Adrian F ; Janthur, Wolf-Dieter ; Waibel, Christine ; Mach, Nicolas ; Froesch, Patrizia ; Buess, Martin ; Bohanes, Pierre ; Godar, Gilles ; Rusterholz, Corinne ; Gonzalez, Michel ; Pless, Miklos

\begin{abstract}
PURPOSE For patients with resectable stage IIIA(N2) non-small-cell lung cancer, neoadjuvant chemotherapy with cisplatin and docetaxel followed by surgery resulted in a 1-year event-free survival (EFS) rate of $48 \%$ in the SAKK $16 / 00$ trial and is an accepted standard of care. We investigated the additional benefit of perioperative treatment with durvalumab. METHODS Neoadjuvant treatment consisted of three cycles of cisplatin $100 \mathrm{mg} / \mathrm{m}^{2}$ and docetaxel $85 \mathrm{mg} / \mathrm{m}^{2}$ once every 3 weeks followed by two doses of durvalumab $750 \mathrm{mg}$ once every 2 weeks. Durvalumab was continued for 1 year after surgery. The primary end point was 1-year EFS. The hypothesis for statistical considerations was an improvement of 1-year EFS from $48 \%$ to $65 \%$. RESULTS Sixty-eight patients were enrolled, 67 were included in the full analysis set. Radiographic response rate was $43 \%$ (95\% CI, 31 to 56) after neoadjuvant chemotherapy and $58 \%$ (95\% CI, 45 to 71) after sequential neoadjuvant immunotherapy. Fifty-five patients were resected, of which $34(62 \%)$ achieved a major pathologic response (MPR; $10 \%$ viable tumor cells) and 10 (18\%) among them a complete pathologic response. Postoperative nodal downstaging (ypN0-1) was observed in 37 patients $(67 \%)$. Fifty-one (93\%) resected patients had an R0 resection. There was no significant effect of pretreatment PD-L1 expression on MPR or nodal downstaging. The 1-year EFS rate was $73 \%$ (twosided $90 \%$ CI, 63 to 82 ). Median EFS and overall survival were not reached after 28.6 months of median follow-up. Fifty-nine (88\%) patients had an adverse event grade 3 including two fatal adverse events that were judged not to be treatment-related. CONCLUSION The addition of perioperative durvalumab to neoadjuvant chemotherapy in patients with stage IIIA(N2) non-small-cell lung cancer is safe and exceeds historical data of chemotherapy alone with a high MPR and an encouraging 1-year EFS rate of $73 \%$.
\end{abstract}

DOI: https://doi.org/10.1200/JCO.21.00276

Posted at the Zurich Open Repository and Archive, University of Zurich

ZORA URL: https://doi.org/10.5167/uzh-207119

Journal Article

Published Version

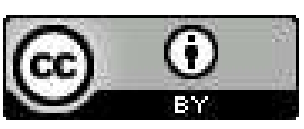

The following work is licensed under a Creative Commons: Attribution 4.0 International (CC BY 4.0) License. 
Originally published at:

Rothschild, Sacha I; Zippelius, Alfred; Eboulet, Eric I; Savic Prince, Spasenija; Betticher, Daniel; Bettini, Adrienne; Früh, Martin; Joerger, Markus; Lardinois, Didier; Gelpke, Hans; Mauti, Laetitia A; Britschgi, Christian; Weder, Walter; Peters, Solange; Mark, Michael; Cathomas, Richard; Ochsenbein, Adrian F; Janthur, Wolf-Dieter; Waibel, Christine; Mach, Nicolas; Froesch, Patrizia; Buess, Martin; Bohanes, Pierre; Godar, Gilles; Rusterholz, Corinne; Gonzalez, Michel; Pless, Miklos (2021). SAKK 16/14: Durvalumab in Addition to Neoadjuvant Chemotherapy in Patients With Stage IIIA(N2) Non-Small-Cell Lung Cancer-A Multicenter Single-Arm Phase II Trial. Journal of Clinical Oncology, 39(26):2872-2880. DOI: https://doi.org/10.1200/JCO.21.00276 


\section{SAKK 16/14: Durvalumab in Addition to Neoadjuvant Chemotherapy in Patients With Stage IIIA(N2) Non-Small-Cell Lung Cancer-A Multicenter Single-Arm Phase II Trial}

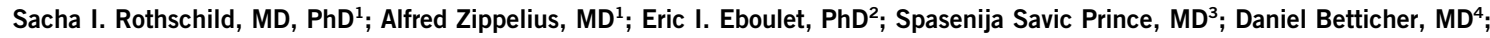
Adrienne Bettini, MD; Martin Früh, MD ${ }^{5,6}$; Markus Joerger, MD, PhD ${ }^{5}$; Didier Lardinois, $\mathrm{MD}^{7}$; Hans Gelpke, $\mathrm{MD}^{8}$; Laetitia A. Mauti, MD, $\mathrm{PhD}^{9}$; Christian Britschgi, MD, PhD ${ }^{10}$; Walter Weder, $\mathrm{MD}^{11}$; Solange Peters, MD, PhD ${ }^{12}$; Michael Mark, $\mathrm{MD}^{13}$; Richard Cathomas, MD ${ }^{13}$; Adrian F. Ochsenbein, MD'; Wolf-Dieter Janthur, MD ${ }^{14}$; Christine Waibel, MD ${ }^{15}$; Nicolas Mach, MD ${ }^{16}$; Patrizia Froesch, $\mathrm{MD}^{17}$; Martin Buess, MD, $\mathrm{PhD}^{18}$; Pierre Bohanes, $\mathrm{MD}^{19}$; Gilles Godar, $\mathrm{MSc}^{2}$; Corinne Rusterholz, $\mathrm{PhD}^{2}$; Michel Gonzalez, $\mathrm{MD}^{20}$; and Miklos Pless, $\mathrm{MD}^{9}$; for the Swiss Group for Clinical Cancer Research (SAKK)

ASSOCIATED CONTENT

See accompanying editorial on page $\mathbf{2 8 5 5}$ Appendix Data Supplement Protocol

Author affiliations and support information (if applicable) appear at the end of this article.

Accepted on June 4, 2021 and published at ascopubs.org/journal/ jco on July 12, 2021: DOI https://doi.org/10. $1200 / J C 0.21 .00276$

PURPOSE For patients with resectable stage IIIA(N2) non-small-cell lung cancer, neoadjuvant chemotherapy with cisplatin and docetaxel followed by surgery resulted in a 1-year event-free survival (EFS) rate of $48 \%$ in the SAKK $16 / 00$ trial and is an accepted standard of care. We investigated the additional benefit of perioperative treatment with durvalumab.

METHODS Neoadjuvant treatment consisted of three cycles of cisplatin $100 \mathrm{mg} / \mathrm{m}^{2}$ and docetaxel $85 \mathrm{mg} / \mathrm{m}^{2}$ once every 3 weeks followed by two doses of durvalumab 750 mg once every 2 weeks. Durvalumab was continued for 1 year after surgery. The primary end point was 1-year EFS. The hypothesis for statistical considerations was an improvement of 1-year EFS from $48 \%$ to $65 \%$.

RESULTS Sixty-eight patients were enrolled, 67 were included in the full analysis set. Radiographic response rate was $43 \%(95 \% \mathrm{Cl}, 31$ to 56$)$ after neoadjuvant chemotherapy and $58 \%(95 \% \mathrm{Cl}, 45$ to 71$)$ after sequential neoadjuvant immunotherapy. Fifty-five patients were resected, of which $34(62 \%)$ achieved a major pathologic response (MPR; $\leq 10 \%$ viable tumor cells) and $10(18 \%)$ among them a complete pathologic response. Postoperative nodal downstaging (ypNO-1) was observed in 37 patients (67\%). Fifty-one (93\%) resected patients had an RO resection. There was no significant effect of pretreatment PD-L1 expression on MPR or nodal downstaging. The 1-year EFS rate was 73\% (two-sided $90 \% \mathrm{Cl}, 63$ to 82). Median EFS and overall survival were not reached after 28.6 months of median follow-up. Fifty-nine (88\%) patients had an adverse event grade $\geq 3$ including two fatal adverse events that were judged not to be treatment-related.

CONCLUSION The addition of perioperative durvalumab to neoadjuvant chemotherapy in patients with stage IIIA(N2) non-small-cell lung cancer is safe and exceeds historical data of chemotherapy alone with a high MPR and an encouraging 1-year EFS rate of $73 \%$.

J Clin Oncol 39:2872-2880. $\odot 2021$ by American Society of Clinical Oncology

\section{INTRODUCTION}

Non-small-cell lung cancer (NSCLC) accounts for $80 \%-85 \%$ of all lung cancers. Approximately $20 \%$ of all patients with NSCLC are diagnosed with stage III NSCLC. Despite the option of curative-intent multimodality treatment in most patients with locally advanced NSCLC, 5-year survival rates are only between $19 \%$ and $36 \% .^{1,2}$ The management of patients with potentially resectable stage IIIA(N2) NSCLC is controversial. Neoadjuvant chemotherapy followed by surgery as well as definitive combined chemoradiotherapy (CRT) are standard treatment options. Several trials have shown that the addition of a second local treatment approach (ie, neoadjuvant or adjuvant radiotherapy, or surgery after $\mathrm{CRT}$ ) does not improve outcome. . $^{3-5}$

The Swiss cooperative group for Cancer Research (SAKK) substantially contributed to establish a standard of care for patients with locally advanced stage III(N2) NSCLC. In the single-arm phase II trial SAKK 16/96, 90 patients with stage IIIA(N2) NSCLC were treated with three cycles of neoadjuvant chemotherapy with cisplatin and docetaxel followed by surgery resulting in a median overall survival (OS) of 27 months. ${ }^{6}$ Complete resection and nodal downstaging were the most relevant prognostic determinants. ${ }^{7}$ The randomized phase 


\section{CONTEXT}

\section{Key Objective}

In patients with resectable stage IIIA(N2) non-small-cell lung cancer (NSCLC), neoadjuvant chemotherapy with cisplatin and docetaxel is an accepted standard of care based on the previous work of our group. The objective of this trial is to demonstrate that the addition of perioperative durvalumab is efficacious and feasible.

\section{Knowledge Generated}

The addition of perioperative durvalumab to neoadjuvant chemotherapy for stage IIIA(N2) NSCLC led to an increase in the 1year event-free survival to $73 \%$ compared with $48 \%$ in a patient population selected with identical inclusion and exclusion criteria but receiving chemotherapy only in our previous trials. In addition, we demonstrated that neoadjuvant use of durvalumab after chemotherapy is safe and well-tolerated and resulted in a high rate of pathologic regression. In contrast to the previously published trials with neoadjuvant immunotherapy, all patients in this study had confirmed involvement of the N2 lymph nodes and thus belong to the highest-risk group of patients for recurrence after surgery.

\section{Relevance}

Our results support the addition of perioperative immune checkpoint inhibitors to neoadjuvant chemotherapy in patients with resectable stage IIIA(N2) NSCLC. The fact that the high pathologic remission rate is associated with overall outcome, which has also been shown in other trials, has implications for the design of future trials.

III trial SAKK 16/00 showed that the addition of sequential neoadjuvant radiotherapy to three cycles of cisplatin and docetaxel did not improve OS. ${ }^{3}$ A pooled analysis of three SAKK trials including more than 350 patients with resectable stage III NSCLC treated with neoadjuvant chemotherapy with cisplatin and docetaxel showed very encouraging 5 - and 10year survival rates (38\% and $28 \%$, respectively) for stage IIIA NSCLC. ${ }^{8}$ Furthermore, surgical mortality was low with 30-day mortality of $0.5 \%$ for lobectomies and $5.3 \%$ for pneumonectomies, including multiple extended resections. On the basis of these results, induction chemotherapy with cisplatin and docetaxel for three cycles followed by tumor resection is now an accepted standard of care.

Inhibitors of programmed cell death 1 (PD-1) and programmed cell death-ligand 1 (PD-L1) have recently changed the treatment of metastatic NSCLC. ${ }^{9}$ In patients with unresectable stage III NSCLC, consolidation therapy with durvalumab after definitive CRT improves OS compared with placebo. ${ }^{10}$ At least four large randomized phase III trials of adjuvant PD-1 and PD-L1 blockade in completely resected patients with stage IB-IIIA have been launched, but have not reported outcomes yet. Preclinical data suggest that neoadjuvant immunotherapy may be more effective than adjuvant therapy. ${ }^{11}$ Antigen presentation and anti-PD-1and anti-PD-L1-facilitated T-cell (re)activation and expansion might be more likely to occur in the microenvironment of a macroscopic tumor than in micrometastases. Initial trials investigating the use of PD-1 and PD-L1 inhibitors in the neoadjuvant setting showed a rate of major pathologic response (MPR) of $19 \%-45 \%$ in patients with stage I-III NSCLC, with $5 \%-15 \%$ of patients achieving a pathologic complete response ( $p C R$ ). ${ }^{12-17}$ The combination of neoadjuvant chemotherapy with PD-1 and PD-L1 inhibitors resulted in an MPR rate of 57\%-84\% and a pCR rate of 33\%-
$59 \% .{ }^{18,19}$ Despite these promising results, there is still a debate on the use of neoadjuvant immune checkpoint inhibitors, especially regarding their toxicity, the delay in surgery and the danger of postoperative complications, and the relevance of surrogate end points such as MPR or PCR.

SAKK 16/14 investigates the efficacy and safety of sequential chemotherapy with cisplatin and docetaxel and immunotherapy with the anti-PD-L1 antibody durvalumab before surgery followed by adjuvant durvalumab in patients with stage IIIA(N2) NSCLC.

\section{METHODS}

\section{Study Design and Participants}

This investigator-initiated, open-label, multicenter, singlearm, phase II trial was performed at 14 sites within the SAKK network in Switzerland (Data Supplement, online only).

Patients were eligible for enrollment if they were between 18 and 75 years with an Eastern Cooperative Oncology Group performance status score of $0-1$ and had a pathologically proven, locally advanced T1-3N2MO, stage IIIA(N2) NSCLC, according to the seventh edition of the TNM classification. Staging was done by positron emission tomography-computed tomography (PET-CT) and brain MRI. The N2 involvement had to be proven by mediastinoscopy or endobronchial ultrasonography with transbronchial fine-needle aspiration. Resectable N2 was not limited to a single station. Details for mediastinal lymph node staging are given in the Protocol (online only) and Data Supplement. The lung function assessment for surgery was performed according to the guidelines of the European Society of Thoracic Surgeons. ${ }^{20}$ Primary technical resectability was assessed by local surgeons with the 
aim to achieve complete resection according to RamiPorta, ${ }^{21}$ and it was validated by an interdisciplinary tumor board. Full inclusion and exclusion criteria are listed in the trial Protocol. This trial is registered with ClinicalTrials.gov (identifier: NCT02572843).

Tumor tissue from initial biopsy and resection specimens underwent central pathology review in accordance with the WHO classification (4th edition, 2015) and the protocol of the College of American Pathologists. ${ }^{22,23}$ Pathologic response was evaluated by assessing the percentage of residual viable tumor volume in relation to the tumor bed. The tumor bed consists of viable tumor, necrosis, and stroma. ${ }^{24,25}$ MPR was defined as $\leq 10 \%$ viable tumor cells, whereas pCR was defined as no evidence of viable tumor cells. ${ }^{26}$ PDL1 testing was performed using the Ventana SP263 assay (Ventana, Tucson, AZ; for details, see the Data Supplement).

The trial was done in accordance with the principles of the Declaration of Helsinki. The Protocol was approved by the ethics committee of each participating site. Written informed consent was obtained from all patients.

\section{Treatment}

Chemotherapy consisted of three cycles of $100 \mathrm{mg} / \mathrm{m}^{2}$ cisplatin and $85 \mathrm{mg} / \mathrm{m}^{2}$ docetaxel given once every 3 weeks, with mandatory granulocyte colony-stimulating factor.

Two doses of durvalumab $750 \mathrm{mg}$ were administered sequentially three weeks after the last dose of chemotherapy, two weeks apart. In case of premature discontinuation of chemotherapy, durvalumab treatment started 3-5 weeks after the last dose of chemotherapy. Guidelines for treatment and dose adjustments are described in the Protocol.

Surgery was scheduled 2-4 weeks after the last application of durvalumab. Tumor resection could be performed by thoracotomy or video-assisted thoracoscopic surgery. Surgery included an anatomical resection such as lobectomy or pneumonectomy with a mediastinal lymph node dissection as previously described. ${ }^{27}$ Patients in whom resection was incomplete were allowed to receive postoperative radiotherapy beginning 4-6 weeks after surgery.

Adjuvant durvalumab given at a dose of $750 \mathrm{mg}$ once every two weeks for 26 cycles started between 4 and 6 weeks after surgery.

\section{Assessments}

Standard baseline assessments had to be done within 42 days before inclusion. Details are provided in the Protocol. All patients underwent PET-CT and mediastinal lymph node staging within 30 days before inclusion.

Restaging (PET-CT) was performed after cycle three of chemotherapy and again within 2 weeks before surgery. Blood and stool samples were taken serially; the details are described in the Protocol.
Lifelong follow-up visits were planned 1 month after surgery, then every 3 months for 2 years, every 6 months in years 3-5, and then every 12 months.

Tumor assessments were performed locally by the study centers and reported according to RECIST version 1.1. Morbidity, mortality, and surgical complications were monitored during the first 30 days after surgery.

Adverse events (AEs) and abnormal laboratory findings were graded according to the National Cancer Institute Common Terminology Criteria for Adverse Events ( $\mathrm{NCl}-$ CTCAE) version 4.03.

\section{End Points}

The primary end point was event-free survival (EFS) at 1 year. An event was defined as relapse or progression according to RECIST 1.1 criteria, secondary tumor, or death because of any cause, whichever occurred first. Secondary end points were EFS, OS, objective response rate (ORR) after neoadjuvant chemotherapy and after neoadjuvant chemotherapy and immunotherapy, pCR, MPR, rate of nodal downstaging to $<$ ypN2, complete resection (RO), pattern of recurrence, AEs, and postoperative 30-day mortality. Definitions of the end points are available in the Data Supplement. Details for prespecified exploratory end points are given in the Protocol.

\section{Statistical Analysis}

A 1-year EFS rate $\leq 48 \%$ (on the basis of the primary analysis of trial SAKK $16 / 00)^{3}$ was considered not promising, whereas a rate $\geq 65 \%$ was considered promising. According to a single-stage phase II design on the basis of survival rate at a specific time point, 64 patients were needed to obtain a power of $80 \%$ with a significance level of $5 \%$. Assuming a $5 \%$ rate of nonevaluable patients, the target sample size was set at 68 patients.

An interim safety analysis was performed after the first 25 patients, with the possibility of an extended examination of the data by an independent data-monitoring committee in case of a postoperative 30-day mortality rate $>10 \%$.

All efficacy end points were analyzed on the basis of the full analysis set (FAS: eligible patients who received at least one dose of chemotherapy). Toxic effects were assessed in the safety population, which comprised patients who received at least one dose of chemotherapy. FAS-2 was defined as all patients who received at least one dose of chemotherapy and at least one dose of durvalumab.

We used the Kaplan-Meier method to estimate EFS and OS. Survival curves and rates were compared using the logrank test and Kaplan-Meier method at a specific time point, and the $\mathrm{Cl}$ was estimated on the basis of the log-log transformation of the Kaplan Meier estimator. Hazard ratios and odds ratios and their $95 \% \mathrm{Cls}$ were calculated with the Cox regression model and logistic regression to explore the association between possible prognostic factors and 
TABLE 1. Baseline Demographic and Clinical Characteristics of the FAS Population

\begin{tabular}{lc} 
Characteristic & Patients $(\mathbf{n}=\mathbf{6 7})$ \\
\hline Age, years (range) & $61(41-74)$ \\
\hline Sex, No. (\%) & $35(52)$ \\
\hline Male & $32(48)$ \\
\hline Female & \\
\hline ECOG performance status, No. (\%) & $52(78)$ \\
\hline 0 & $15(22)$ \\
\hline 1 & \\
\hline Smoking status, No. (\%) & $28(42)$ \\
\hline Current & $36(54)$ \\
\hline Former & $3(5)$ \\
\hline Never & $37(55)$ \\
\hline Histology, No. (\%) & $22(33)$ \\
\hline Adenocarcinoma & $7(10)$ \\
\hline Squamous cell carcinoma & $1(2)$ \\
\hline NSCLC NOS & $15(22)$ \\
\hline Large-cell neuroendocrine carcinoma & $33(49)$ \\
\hline T at presentation, No. (\%) & $19(28)$ \\
\hline T1 & \\
\hline T2 & \\
\hline T3 & \\
\hline
\end{tabular}

Abbreviations: ECOG, Eastern Cooperative Oncology Group; FAS, full analysis set; NOS, not otherwise specified; NSCLC, non-small-cell lung cancer; $\mathrm{T}$, tumor stage.

EFS, OS, pCR, MPR, and ypN. Statistical significance was set at $P$ value $<.05$. SAS 9.4 (SAS Institute Inc) and $\mathrm{R}$ v3.5.1 were used for analyses.

\section{RESULTS}

Between June 16, 2016, and January 16, 2019, 68 patients were enrolled. Sixty-seven patients fulfilled the FAS and patient disposition is shown in the CONSORT diagram (Data Supplement). One patient did not receive neoadjuvant chemotherapy because of a brain metastasis reported 3 days after registration. Baseline characteristics are presented in Table 1. Thirty-two (48\%) of the pretreatment tumor samples showed a PD-L1 expression in $\geq 1 \%$ and $13(19 \%)$ in $\geq 25 \%$ of tumor cells (high PD-L1 expression; Appendix Table A1, online only).

Sixty (90\%) of 67 patients completed three cycles of neoadjuvant chemotherapy. The median relative total dose intensity of chemotherapy was above 97\% (Data Supplement). Total dose administration is shown in the Data Supplement. Twenty-two patients (33\%) received carboplatin instead of cisplatin during the second and third ( $n=15)$ or third cycle $(n=7)$ of neoadjuvant chemotherapy, mainly because of renal toxicity or hearing impairment. Sixty-two (93\%) of the 67 patients started neoadjuvant durvalumab treatment. Fifty-five (82\%) patients underwent tumor resection. The most common reasons patients were not operated on were disease progression $(n=6)$, treatment discontinuation because of toxicity $(n=3)$, and inoperability $(n=3)$. Fifty $(75 \%)$ patients started adjuvant durvalumab treatment. Median duration of adjuvant durvalumab treatment was 52 weeks (range, 2-54).

Of the 55 patients undergoing resection, $43(78 \%)$ had a lobectomy, seven (13\%) a bilobectomy, and five (9\%) a pneumonectomy. The overall 30-day postoperative mortality was $2 \%$ (one patient with fatal bronchopulmonary bleeding, which was deemed unrelated to the study treatment). In 51 of 55 patients (93\%) an RO surgical resection was achieved, three (6\%) patients had an $\mathrm{R} 1$, and one (2\%) patient an $\mathrm{R} 2$ resection. Six (11\%) patients underwent postoperative radiotherapy. The reasons were incomplete resection in four patients and investigator decision against the Protocol recommendation in two patients.

At the time of data cutoff (July 10, 2020), 25 (37\%) patients had completed the trial treatment as per Protocol, 37 patients had discontinued the trial treatment, and five patients were still on trial treatment. With a median followup of 28.6 months (range: 2.1-47.9), 45 (67\%) patients were alive and free of recurrence. Twenty-two (33\%) of 67 patients in the FAS population had disease progression or had died. In the FAS, 1-year EFS was 73\% (two-sided 90\% $\mathrm{Cl}, 63$ to 82). Therefore, the primary end point of the trial was reached. Median EFS was not reached (Fig 1). Twoyear EFS was $68 \%$ (95\% Cl, 54 to 78). Median OS was not reached (Fig 2). OS rates at 1 and 2 years were 91\% (95\% $\mathrm{Cl}, 81$ to 96$)$ and $83 \%(95 \% \mathrm{Cl}, 71$ to 90$)$. Overall, 15 (22\%) patients died. Causes of death were progressive disease in 12 (80\%) patients, bronchopulmonary bleeding after surgery in one patient, sepsis after surgery in one patient, and pulmonary embolism after completing adjuvant durvalumab in one patient.

After neoadjuvant chemotherapy, the ORR according to RECIST 1.1 was $43 \%$ (29 of 67; 95\% Cl, 31 to 56), including two (3\%) patients with a CR and 27 (40\%) patients with a PR (Appendix Table A2, online only; Fig 3). After neoadjuvant durvalumab ( $n=62$ ), ORR was $58 \%$ (36 of $62 ; 95 \% \mathrm{Cl}, 45$ to 71 ), with four (7\%) and 32 (52\%) patients achieving a CR and PR, respectively. Overall, seven (11\%) patients had disease progression during neoadjuvant therapy.

Thirty-four of 55 patients $(62 \%$; $95 \% \mathrm{Cl} 48-75)$ achieved an MPR (Table 2, Fig 3). Among them were 10 patients with a pCR (10 of 55 patients, $18 \%$; $95 \% \mathrm{Cl}, 9$ to 31). Seven (70\%) of 10 patients with a pCR and 19 (56\%) of 34 patients with an MPR had a radiographic complete or partial response after neoadjuvant therapy (Data Supplement). Radiographic response was associated with pathologic response $(r=0.399 ; 95 \% \mathrm{Cl}, 0.136$ to 0.609 ; 


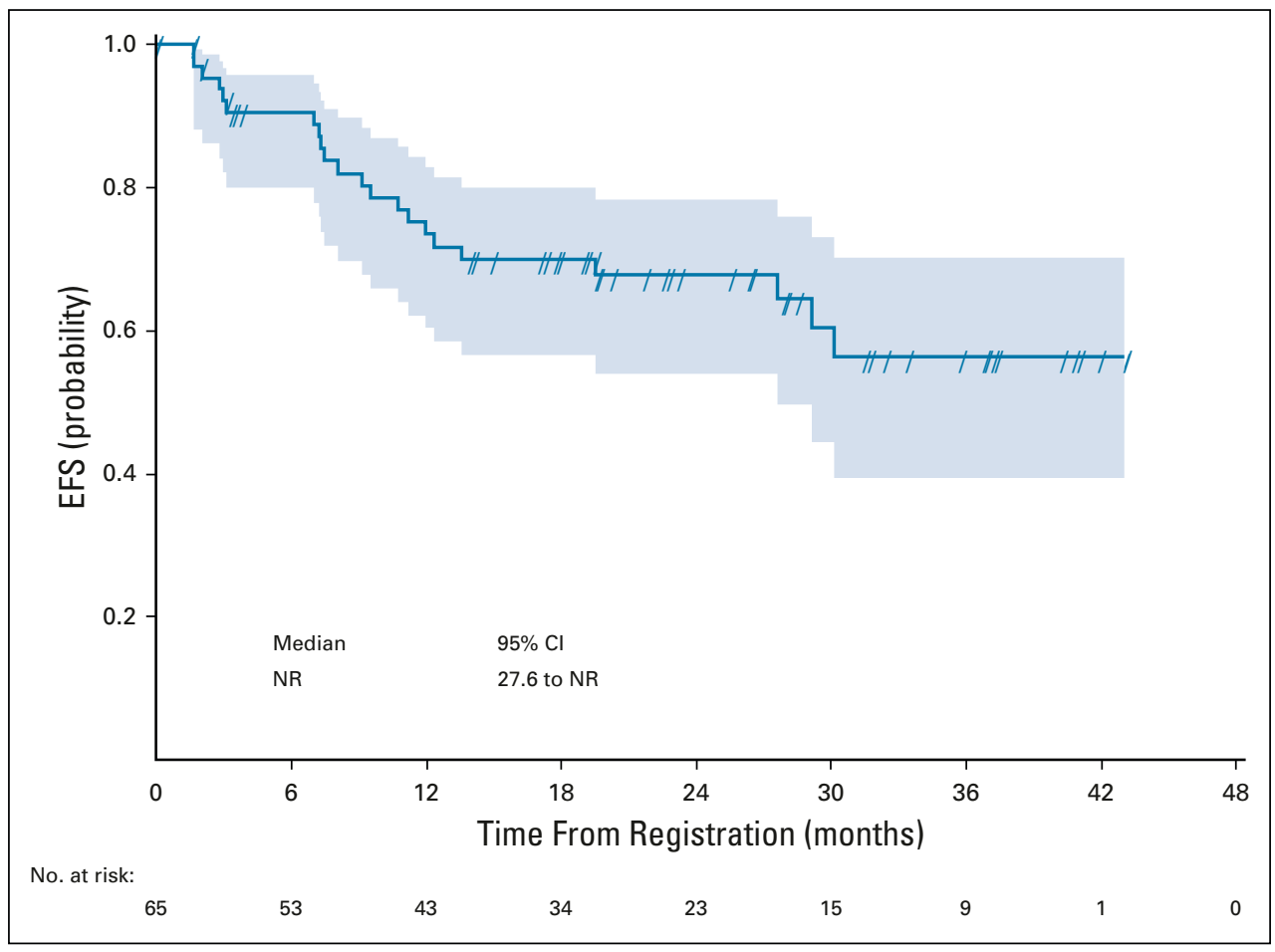

FIG 1. Kaplan-Meier survival curve for EFS. Dotted lines represent $95 \% \mathrm{Cls}$. EFS, event-free survival; NR, not reached.

$P=.004 ;$ Data Supplement). Nodal downstaging to ypN1 occurred in $11(20 \%)$ of 55 patients, whereas ypNO was found in $26(47 \%)$ of 55 patients. Patients with a PD-L1 expression of $\geq 25 \%$ had a higher rate of $\mathrm{pCR}$ (odds ratio, 4.8; $95 \% \mathrm{Cl}, 1.0$ to 22.8; $P=.047$; Data Supplement). However, PD-L1 expression was not significantly

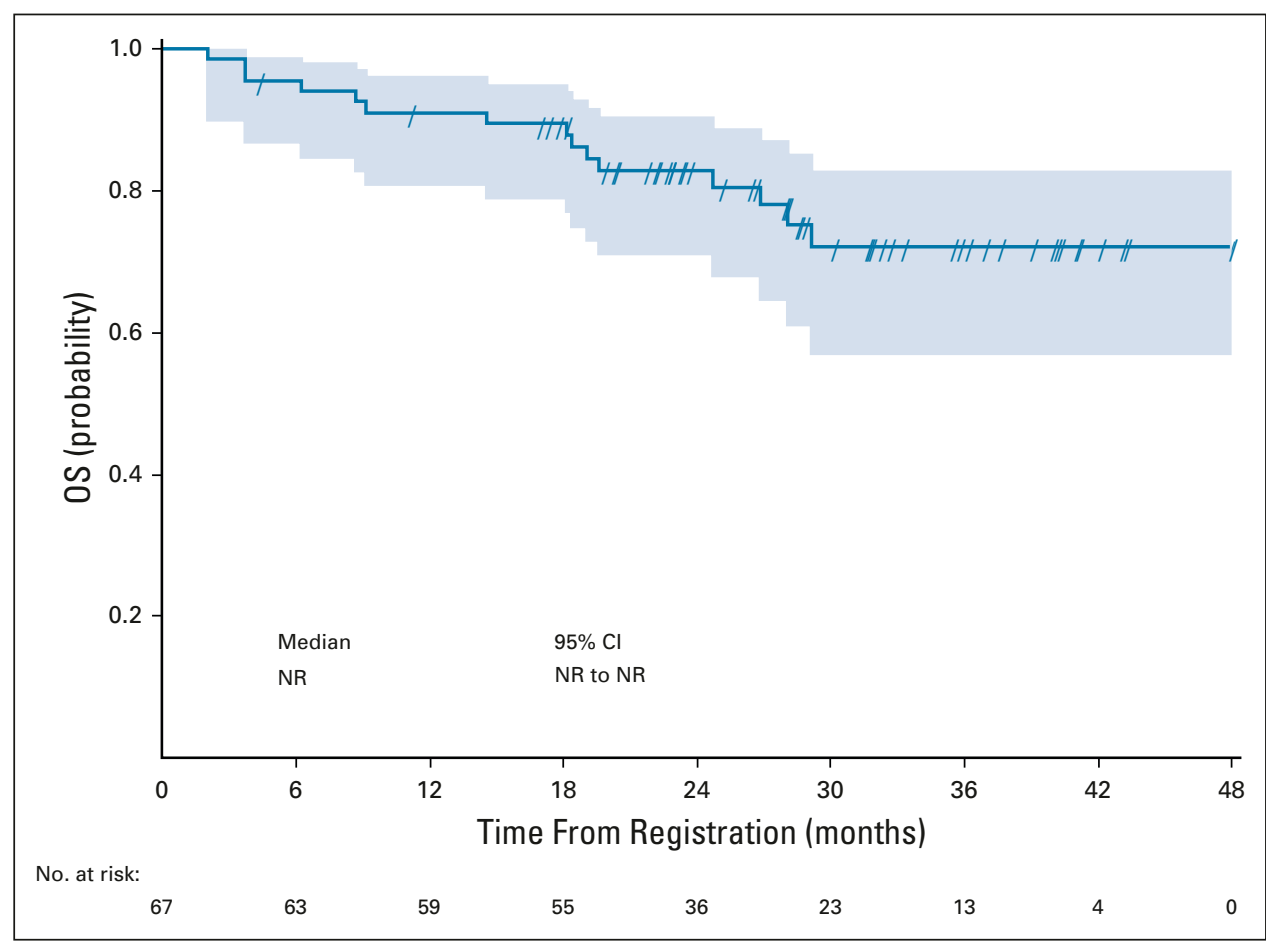

FIG 2. Kaplan-Meier survival curve for OS. Dotted lines represent $95 \%$ Cls. NR, not reached; OS, overall survival. 


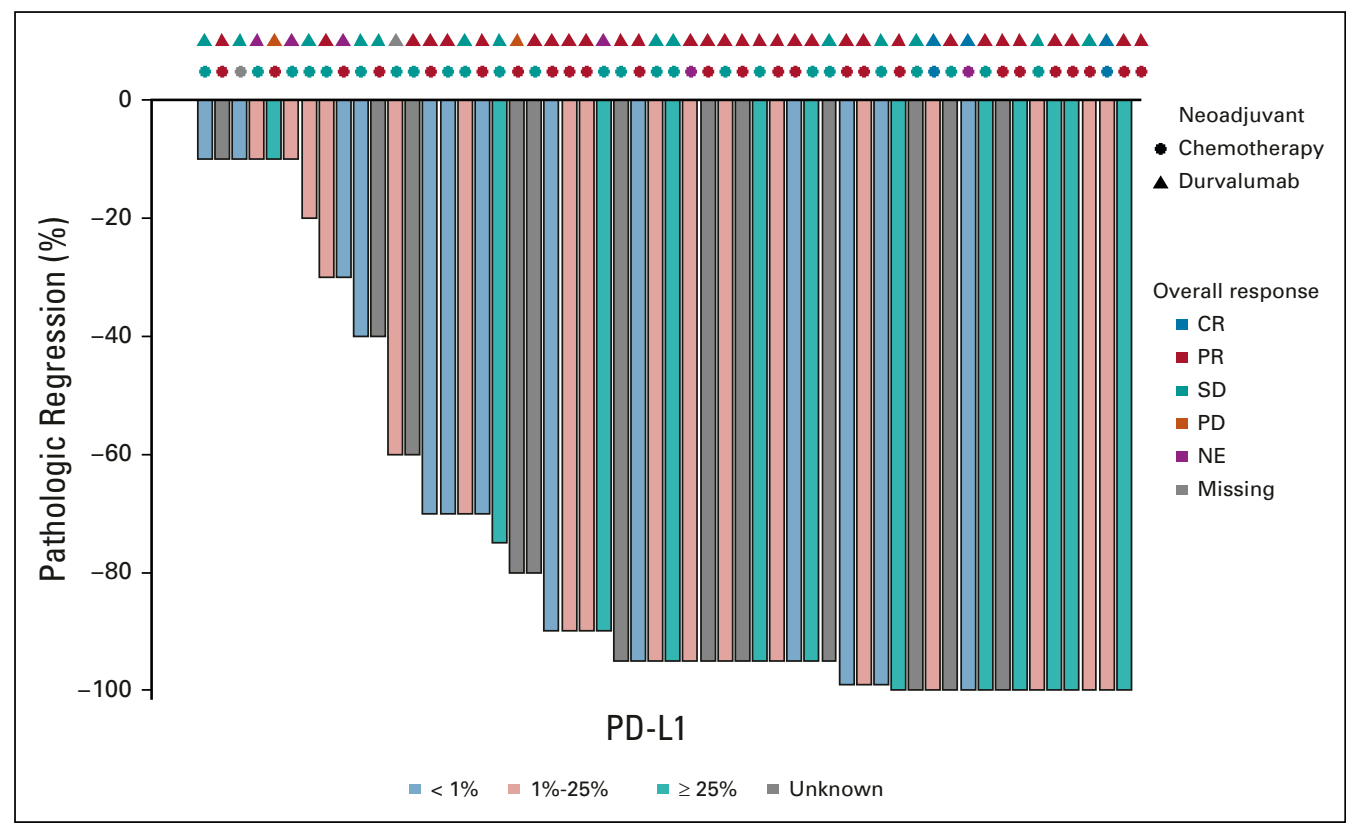

FIG 3. Waterfall plot. Only data from patients who underwent resection are included $(n=55)$. One patient underwent lung wedge resection at diagnosis (UPN 063). Therefore, pathologic regression could not be assessed in this patient. Pathologic regression is defined as percent residual viable tumor volume in relation to the tumor bed. The tumor bed consists of viable tumor, necrosis, and stroma. CR, complete remission; NE, not evaluable; PD, programmed cell death 1; PD-L1, programmed cell death-ligand 1; PR, partial remission; SD, stable disease.

associated with MPR, nodal downstaging, or EFS at 1-year (Data Supplement).

Disease progression after resection occurred in 15 (27\%) of 55 patients, three of whom had achieved an MPR and two ypNO. The pattern of recurrence is shown in the Data Supplement. Of the seven patients with a local recurrence, four underwent salvage radiotherapy. In a post hoc analysis, median EFS was significantly longer for patients achieving an MPR or pCR; likewise, MPR also significantly predicted OS (Data Supplement). Nodal clearance (ypNO) was significantly associated with a longer median EFS (Data Supplement). Of the 12 patients who did not undergo tumor resection, four (33\%) patients had disease progression and six (50\%) have died.

All 67 patients included in the safety population experienced at least one AE. In total, 59 (88\%) patients had an AE grade $\geq 3$ including two fatal AEs. One patient died because of respiratory failure during neoadjuvant

TABLE 2. Pathologic Response

\begin{tabular}{lcc} 
Response & Total $(\mathbf{n}=\mathbf{5 5})$, No. (\%) & $\mathbf{9 5 \% ~} \mathbf{~ I ~}$ \\
\hline pCR & $10(18)$ & 9 to 31 \\
\hline MPR $^{a}$ & $34(62)$ & 48 to 75 \\
\hline Nodal downstaging & $37(67)$ & 53 to 79 \\
\hline$y p N 0$ & $26(47)$ & \\
\hline$y p N 1$ & $11(20)$ &
\end{tabular}

Abbreviations: MPR, major pathologic response; pCR, pathologic complete response; yp, postoperative staging after neoadjuvant therapy aDefined as $\leq 10 \%$ viable tumor cells. chemotherapy, most likely caused by disease progression. Another patient died because of bronchopulmonary hemorrhage 10 days after surgery. Regarding each phase of the trial treatment, all patients (grade $\geq 3$ : $67 \%$ ) experienced an AE during neoadjuvant chemotherapy, 50 ( $81 \%$; grade $\geq 3: 13 \%$ ) patients during neoadjuvant immunotherapy, 48 ( $87 \%$; grade $\geq 3: 29 \%$ ) patients during the perioperative phase, and 49 (98\%; grade $\geq 3: 50 \%)$ patients during adjuvant immunotherapy (Appendix Table A3, online only; Table 3; Data Supplement). AEs of special interest with durvalumab were pneumonitis in two (3\%), hypersensitivity reactions in three $(5 \%)$, and hepatic function abnormalities in six (10\%) patients.

\section{DISCUSSION}

To our knowledge, this is thus far the largest reported prospective trial of perioperative anti-PD-L1 therapy in addition to neoadjuvant chemotherapy in patients with resectable NSCLC. Furthermore, to our knowledge, it is the first study focusing on patients with stage IIIA(N2) disease with confirmed mediastinal lymph node involvement. We recently showed long-term survival data of $29 \%$ after 10 years for patients with stage IIIA(N2) NSCLC treated with neoadjuvant chemotherapy. ${ }^{8}$ In this trial, we investigated the addition of perioperative durvalumab to standard platinum-based neoadjuvant chemotherapy. The goal of improving EFS from $48 \%$ to $65 \%$ after one year was clearly achieved with an EFS at one year of $73 \%$. After a median follow-up period of 28.6 months, neither the median EFS 
TABLE 3. Treatment-Related AEs With Durvalumab in the Neoadjuvant Setting $(n=62)$

\begin{tabular}{|c|c|c|c|}
\hline AE & Grades 1 and 2 & Grade 3 & Grade 4 \\
\hline Adrenal insufficiency & & $1(2)$ & \\
\hline ALT increased & $1(2)$ & $3(5)$ & \\
\hline Arthralgia & $8(13)$ & & \\
\hline Arthritis & $1(2)$ & $1(2)$ & \\
\hline AST increased & $2(3)$ & $1(2)$ & \\
\hline Autoimmune hepatitis & & $1(2)$ & \\
\hline Diarrhea & $11(18)$ & $1(2)$ & \\
\hline Dyspnea & & $3(5)$ & \\
\hline Fatigue & $21(34)$ & $3(5)$ & \\
\hline Hyperkeratosis & & $1(2)$ & \\
\hline Hyperthyroidism & $6(10)$ & & \\
\hline Lipase increased & & & $1(2)$ \\
\hline Lung infection & $1(2)$ & $3(5)$ & \\
\hline Nausea & $8(13)$ & $1(2)$ & \\
\hline Pneumonitis & $1(2)$ & $1(2)$ & \\
\hline Pruritus & $9(15)$ & $1(2)$ & \\
\hline Rash & $9(15)$ & & \\
\hline
\end{tabular}

NOTE. Data are presented as No. (\%). Shown are the treatment-related AEs of any grade that occurred in more than $10 \%$ of patients or any treatment-related AEs of grade 3 or higher. No grade 5 treatment-related AEs were observed.

Abbreviation: $\mathrm{AE}$, adverse event.

nor the median OS was reached. The surgical 30-day postoperative mortality rate was low and comparable with previous trials of our group with neoadjuvant chemotherapy alone. $^{8}$

Early tumor progression and immune-mediated toxicities under immune checkpoint inhibition are major concerns when using this new treatment strategy. In our trial, $82 \%$ of the patients were operated. This number is consistent with our previous data with chemotherapy alone ${ }^{3,6}$ and in agreement with other trials in patients with stage IIIA NSCLC after neoadjuvant treatment. ${ }^{28}$ The most common reason for not operating after neoadjuvant treatment was disease progression. Definitive CRT and additive durvalumab on the basis of the PACIFIC trial ${ }^{10}$ would most likely not have prevented distant metastases and would therefore not have improved the outcome of those patients. Only one patient experienced toxicity that precluded subsequent surgery. One recent trial evaluating 6 weeks of neoadjuvant nivolumab and ipilimumab treatment was terminated early after inclusion of nine patients only because of a high rate of treatment-related AEs grade $\geq 3$ and 3 patients with biopsy-confirmed tumor progression precluding surgery, ${ }^{29}$ whereas in the NEOSTAR trial, 17 of $21(81 \%)$ patients receiving the same combination regimen went on to surgery as planned. ${ }^{14}$
Initial trials investigating the use of PD-1 and PD-L1 inhibitors in the neoadjuvant setting as monotherapy showed a rate of MPR of $19 \%-45 \%$ in patients with stage I-III NSCLC with 5\%-15\% of patients achieving complete pathologic remission. ${ }^{12,13,15-17,30}$ The combination of chemotherapy and immunotherapy as neoadjuvant treatment leads to a further increase in pathologic regression rates, as demonstrated in our trial (MPR 62\% including $18 \%$ pCR). This rate is also clearly higher than the MPR rate in patients treated with neoadjuvant chemotherapy alone, which was below $30 \%$ in our previous trials. ${ }^{6}$ High pathologic regression rates were also shown in two previously published trials with combined neoadjuvant chemotherapy and immunotherapy. The NADIM trial included only stage IIIA patients and demonstrated an MPR rate of $83 \%$ and a 2-year PFS of $77.1 \%$ in resected patients with the combination of carboplatin, paclitaxel, and nivolumab. ${ }^{18}$ In the trial by Shu et al, combined chemoimmunotherapy with atezolizumab in patients with stage IB-IIIA NSCLC resulted in an MPR rate of $57 \%$ and a pCR rate of $33 \%$. In the latter trial, patients who achieved an MPR also had a numerically longer disease-free survival according to a post hoc analysis. ${ }^{19}$ Similarly, a post hoc analysis of our data showed a correlation between MPR and improved EFS as well as OS. Although pathologic regression is a well-established predictor of EFS and OS with neoadjuvant chemotherapy, ${ }^{26}$ its value for patients treated with immune checkpoint inhibitors has not yet been proven. Our trial and other recently published data ${ }^{19}$ seem to support the relevance of MPR and will contribute to the discussion around adequate surrogate end points for neoadjuvant trials and might influence the design of future trials.

Mediastinal lymph node clearance in patients with confirmed mediastinal lymph node involvement is one of the most important prognostic factors after neoadjuvant chemotherapy. In our trial, almost half of the patients achieved nodal clearance, which was a relevant predictor for EFS and OS. Similar findings were observed in other trials investigating combined chemoimmunotherapy. ${ }^{18,19}$ In our trial, pathologic responses were observed independently of tumor PD-L1 expression confirming results from other neoadjuvant trials with immune checkpoint inhibitors. ${ }^{18,19}$

The clinical value of an immunotherapy in early-stage NSCLC has not been clarified yet. Several randomized trials are currently investigating their use in the adjuvant and neoadjuvant setting. As in the NADIM trial, patients in our trial received adjuvant immunotherapy for one year postoperatively. This was not the case in other neoadjuvant trials. The role of adjuvant immune checkpoint inhibitors is currently evaluated in several randomized phase III trials.

Our trial includes a comprehensive translational research program for which we collected serially blood and stool 
samples. These projects are still ongoing and may provide further insights into the activity of perioperative immunotherapy in stage IIIA(N2) NSCLC.

In summary, the SAKK 16/14 trial is the largest published trial of perioperative anti-PD-L1 therapy in addition to neoadjuvant chemotherapy in patients with resectable

\section{AFFILIATIONS}

${ }^{1}$ Department of Medical Oncology and Comprehensive Cancer Center, University Hospital Basel, Basel, Switzerland

${ }^{2}$ SAKK Coordinating Center, Bern, Switzerland

${ }^{3}$ Pathology, Institute of Medical Genetics and Pathology, University Hospital Basel, Basel, Switzerland

${ }^{4}$ Department of Oncology, HFR Fribourg-Hôpital fribourgeois, Fribourg, Switzerland

${ }^{5}$ Department of Oncology/Hematology, Cantonal Hospital St Gallen, St Gallen, Switzerland

${ }^{6}$ Department of Oncology, Inselspital Bern, Bern, Switzerland

${ }^{7}$ Division of Thoracic Surgery, University Hospital Basel, Basel, Switzerland

${ }^{8}$ Department of Thoracic and Visceral Surgery, Cantonal Hospital Winterthur, Winterthur, Switzerland

${ }^{9}$ Department of Oncology, Cantonal Hospital Winterthur, Winterthur, Switzerland

${ }^{10}$ Department of Medical Oncology and Hematology, Comprehensive Cancer Center Zurich, University Hospital Zurich, University of Zurich, Zurich, Switzerland

${ }^{11}$ Department of Thoracic Surgery, University Hospital of Zurich, Zurich, Switzerland

${ }^{12}$ Department of Oncology, University Hospital Lausanne CHUV, Lausanne, Switzerland

${ }^{13}$ Divison of Oncology/Hematology, Cantonal Hospital Graubünden, Chur, Switzerland

${ }^{14}$ Department of Oncology/Hematology, Cantonal Hospital Aarau, Aarau, Switzerland

${ }^{15}$ Department of Oncology, Cantonal Hospital Baden, Baden, Switzerland ${ }^{16}$ Department of Oncology, University Hospital Geneva, Geneva, Switzerland

${ }^{17}$ Oncology Institute of Southern Switzerland, Bellinzona, Switzerland

${ }^{18}$ Division of Medical Oncology, St Claraspital, Basel, Switzerland

${ }^{19}$ Centre de Chimiothérapie Anti-Cancéreuse, Lausanne, Switzerland

${ }^{20}$ Department of Thoracic Surgery, University Hospital Lausanne CHUV, Lausanne, Switzerland

\section{CORRESPONDING AUTHOR}

Sacha I. Rothschild, MD, PhD, Department of Medical Oncology and Comprehensive Cancer Center, University Hospital Basel, Petersgraben 4, 4031 Basel, Switzerland; e-mail: sacha.rothschild@usb.ch.

\section{DISCLAIMER}

The trial was designed by the sponsor and the investigators. The study funders had no role in trial design, data collection, data analysis, data interpretation, or writing of the report. The corresponding author had full access to all the data in the trial and had final responsibility for the decision to submit the manuscript for publication. stage IIIA(N2) NSCLC. The treatment resulted in a high 1year EFS rate of $73 \%$. Our results show that the addition of perioperative durvalumab to neoadjuvant chemotherapy with cisplatin and docetaxel in patients with resectable stage IIIA(N2) NSCLC is a highly active and safe therapy that needs to be further investigated.

\section{PRIOR PRESENTATION}

Presented at the 2020 ASCO Annual Meeting (Virtual), May 29-June 2, 2020 (abstr 9016) and the 2020 European Society for Medical Oncology Congress (Virtual), September 19-21, 2020 (abstr 1237MO).

\section{SUPPORT}

Supported by research agreements with the following institutions: Swiss State Secretary for Education, Research and Innovation (SERI), Swiss Cancer Research Foundation (SCS), and Swiss Cancer League (SCL). Research funding for translational research projects was granted by Gateway for Cancer Research and Rising Tide Foundation. AstraZeneca provided drug and financial support.

\section{CLINICAL TRIAL INFORMATION \\ NCT02572843}

\section{AUTHORS' DISCLOSURES OF POTENTIAL CONFLICTS OF INTEREST}

Disclosures provided by the authors are available with this article at DOI https://doi.org/10.1200/JC0.21.00276.

\section{DATA SHARING STATEMENT}

The trial Protocol did not include a data sharing plan. Therefore, data from the trial will not be shared publicly as data sharing was not included when ethical approval was requested. The full trial Protocol is available with this article at DOI: https://doi.org/10.1200/JC0.21.00276.

\section{AUTHOR CONTRIBUTIONS}

Conception and design: Sacha I. Rothschild, Alfred Zippelius, Eric I. Eboulet, Walter Weder, Michel Gonzalez, Miklos Pless

Provision of study materials or patients: Sacha I. Rothschild, Alfred Zippelius, Spasenija Savic Prince, Daniel Betticher, Adrienne Bettini, Martin Früh, Markus Joerger, Didier Lardinois, Hans Gelpke, Laetitia A Mauti, Christian Britschgi, Walter Weder, Solange Peters, Michael Mark, Richard Cathomas, Adrian F. Ochsenbein, Wolf-Dieter Janthur, Christine Waibel, Nicolas Mach, Patrizia Froesch, Martin Buess, Pierre Bohanes, Michel Gonzalez, Miklos Pless

Collection and assembly of data: Sacha I. Rothschild, Eric I. Eboulet, Spasenija Savic Prince, Daniel Betticher, Adrienne Bettini, Martin Früh, Markus Joerger, Didier Lardinois, Christian Britschgi, Solange Peters, Michael Mark, Richard Cathomas, Adrian F. Ochsenbein, Wolf-Dieter Janthur, Christine Waibel, Nicolas Mach, Patrizia Froesch, Martin Buess, Pierre Bohanes, Gilles Godar, Corinne Rusterholz, Miklos Pless

Data analysis and interpretation: Sacha I. Rothschild, Alfred Zippelius, Eric I. Eboulet, Spasenija Savic Prince, Miklos Pless

Manuscript writing: All authors

Final approval of manuscript: All authors

Accountable for all aspects of the work: All authors

\section{REFERENCES}

1. Goldstraw P, Chansky K, Crowley J, et al: The IASLC Lung Cancer Staging Project: Proposals for revision of the TNM stage groupings in the forthcoming (eighth) edition of the TNM classification for lung cancer. J Thorac Oncol 11:39-51, 2016 
2. Siegel RL, Miller KD, Jemal A: Cancer statistics, 2020. CA Cancer J Clin 70:7-30, 2020

3. Pless M, Stupp R, Ris H-B, et al: Induction chemoradiation in stage IIIA/N2 non-small-cell lung cancer: A phase 3 randomised trial. Lancet 386:1049-1056, 2015

4. Albain KS, Swann RS, Rusch VW, et al: Radiotherapy plus chemotherapy with or without surgical resection for stage III non-small-cell lung cancer: A phase III randomised controlled trial. Lancet 374:379-386, 2009

5. Le Pechoux C, Pourel N, Barlesi F, et al: An international randomized trial, comparing post-operative conformal radiotherapy (PORT) to no PORT, in patients with completely resected non-small cell lung cancer (NSCLC) and mediastinal N2 involvement. Primary end-point analysis of Lung ART (IFCT-0503). Ann Oncol 31:S1178, 2020

6. Betticher DC, Hsu Schmitz S-FF, Totsch M, et al: Mediastinal lymph node clearance after docetaxel-cisplatin neoadjuvant chemotherapy is prognostic of survival in patients with stage IIIA pN2 non-small-cell lung cancer: A multicenter phase II trial. J Clin Oncol 21:1752-1759, 2003

7. Betticher DC, Hsu Schmitz S-F, Tötsch M, et al: Prognostic factors affecting long-term outcomes in patients with resected stage IIIA pN2 non-small-cell lung cancer: 5-year follow-up of a phase II study. Br J Cancer 94:1099-1106, 2006

8. Früh M, Betticher DC, Stupp R, et al: Multimodal treatment in operable stage III NSCLC: A pooled analysis on long-term results of three SAKK trials (SAKK 16/96, 16/00, and 16/01). J Thorac Oncol 14:115-123, 2019

9. Remon J, Passiglia F, Ahn MJ, et al: Immune checkpoint inhibitors in thoracic malignancies: Review of the existing evidence by an IASLC expert panel and recommendations. J Thorac Oncol 15:914-947, 2020

10. Antonia SJ, Villegas A, Daniel D, et al: Overall survival with durvalumab after chemoradiotherapy in stage III NSCLC. N Engl J Med 379:2342-2350, 2018

11. Liu J, Blake SJ, Yong MCR, et al: Improved efficacy of neoadjuvant compared to adjuvant immunotherapy to eradicate metastatic disease. Cancer Discov 6: 1382-1399, 2016

12. Forde PM, Chaft JE, Smith KN, et al: Neoadjuvant PD-1 blockade in resectable lung cancer. N Engl J Med 378:1976-1986, 2018

13. Kwiatkowski DJ, Rusch VW, Chaft JE, et al: Neoadjuvant atezolizumab in resectable non-small cell lung cancer (NSCLC): Interim analysis and biomarker data from a multicenter study (LCMC3). J Clin Oncol 37, 2019 (suppl; abstr 8503)

14. Cascone T, William WN, Weissferdt A, et al: Neoadjuvant nivolumab or nivolumab plus ipilimumab in operable non-small cell lung cancer: The phase 2 randomized NEOSTAR trial. Nat Med 27:504-514, 2021

15. Wislez M, Mazieres J, Lavole A, et al: Neoadjuvant durvalumab in resectable non-small cell lung cancer (NSCLC): Preliminary results from a multicenter study (IFCT-1601 IONESCO). Ann Oncol 31, 2020 (suppl 4; abstr 12140)

16. Gao S, Li N, Gao S, et al: Neoadjuvant PD-1 inhibitor (sintilimab) in NSCLC. J Thorac Oncol 15:816-826, 2020

17. Ready N, Tong BC, Clarke J, et al: Neoadjuvant pembrolizumab in early stage non-small cell lung cancer (NSCLC): Toxicity, efficacy, and surgical outcomes. J Thorac Oncol 14, 2019 (suppl; abstr P2.04-89)

18. Provencio M, Nadal E, Insa A, et al: Neoadjuvant chemotherapy and nivolumab in resectable non-small-cell lung cancer (NADIM): An open-label, multicentre, single-arm, phase 2 trial. Lancet Oncol 21:1413-1422, 2020

19. Shu CA, Gainor JF, Awad MM, et al: Neoadjuvant atezolizumab and chemotherapy in patients with resectable non-small-cell lung cancer: An open-label, multicentre, single-arm, phase 2 trial. Lancet Oncol 21:786-795, 2020

20. Brunelli A, Charloux A, Bolliger CT, et al: The European Respiratory Society and European Society of Thoracic Surgeons clinical guidelines for evaluating fitness for radical treatment (surgery and chemoradiotherapy) in patients with lung cancer. Eur J Cardiothorac Surg 36:181, 2009

21. Rami-Porta R, Wittekind C, Goldstraw P: Complete resection in lung cancer surgery: Proposed definition. Lung Cancer 49:25-33, 2005

22. Travis WD, Brambilla E, Nicholson AG, et al: The 2015 World Health Organization classification of lung tumors: Impact of genetic, clinical and radiologic advances since the 2004 classification. J Thorac Oncol 10:1243-1260, 2015

23. Schneider F, Butnor KJ, Beasley MB, et al: Protocol for the examination of resection specimens from patients with primary non-small cell carcinoma, small cell carcinoma, or carcinoid tumor of the lung. Arch Pathol Lab Med 133:1552-1559, 2020

24. Junker K, Langner K, Klinke F, et al: Grading of tumor regression in non-small cell lung cancer: Morphology and prognosis. Chest 120:1584-1591, 2001

25. Travis WD, Dacic S, Wistuba I, et al: IASLC multidisciplinary recommendations for pathologic assessment of lung cancer resection specimens after neoadjuvant therapy. J Thorac Oncol 15:709-740, 2020

26. Hellmann MD, Chaft JE, William WN, et al: Pathological response after neoadjuvant chemotherapy in resectable non-small-cell lung cancers: Proposal for the use of major pathological response as a surrogate endpoint. Lancet Oncol 15:e42-e50, 2014

27. Lardinois D, De Leyn P, Van Schil P, et al: ESTS guidelines for intraoperative lymph node staging in non-small cell lung cancer. Eur J Cardiothorac Surg 30: 787-792, 2006

28. Eberhardt WEE, Pöttgen C, Gauler TC, et al: Phase III study of surgery versus definitive concurrent chemoradiotherapy boost in patients with resectable stage IIIA(N2) and selected IIIB non-small-cell lung cancer after induction chemotherapy and concurrent chemoradiotherapy (ESPATUE). J Clin Oncol 33: 4194-4201, 2015

29. Reuss JE, Anagnostou V, Cottrell TR, et al: Neoadjuvant nivolumab plus ipilimumab in resectable non-small cell lung cancer. J Immunother Cancer 8:e001282, 2020

30. Cascone T, William WN, Weissferdt A, et al: Neoadjuvant nivolumab (N) or nivolumab plus ipilimumab (NI) for resectable non-small cell lung cancer (NSCLC): Clinical and correlative results from the NEOSTAR study. J Clin Oncol 37, 2019 (suppl; abstr 8504) 
SAKK 16/14: Durvalumab in Addition to Neoadjuvant Chemotherapy in Patients With Stage IIIA(N2) Non-Small-Cell Lung Cancer-A Multicenter Single-Arm Phase II Trial

The following represents disclosure information provided by authors of this manuscript. All relationships are considered compensated unless otherwise noted. Relationships are self-held unless noted. I = Immediate Family Member, Inst = My Institution. Relationships may not relate to the subject matter of this manuscript. For more information about ASCO's conflict of interest policy, please refer to www.asco.org/rwc or ascopubs.org/jco/authors/author-center.

Open Payments is a public database containing information reported by companies about payments made to US-licensed physicians (Open Payments).

Sacha I. Rothschild

Consulting or Advisory Role: Bristol Myers Squibb, AstraZeneca, Boehringer Ingelheim, Eisai, Roche, Novartis, Merck Serono, MSD Oncology, Pfizer,

Takeda, AbbVie

Research Funding: Boehringer Ingelheim, AstraZeneca, Bristol Myers Squibb, Eisai, Merck Serono, AbbVie

Expert Testimony: Roche, AstraZeneca, Bristol Myers Squibb

Travel, Accommodations, Expenses: Roche Pharma AG, Lilly, Bristol Myers

Squibb, AstraZeneca, Merck Sharp \& Dohme, Amgen

Alfred Zippelius

Honoraria: BMSi, MSD, Roche, NBE Therapeutics, ACM Pharma, Hookipa Biotech, BeyondSpring Pharmaceuticals

Research Funding: Roche, NBE Therapeutics, Secarna, ACM Pharma, Hookipa Biotech, Beyondsprings

\section{Spasenija Savic Prince}

Honoraria: Novartis

Consulting or Advisory Role: Diaceutics Ireland Limited, Merck (Schweiz) AG AstraZeneca AG

\section{Daniel Betticher}

Travel, Accommodations, Expenses: Bayer

\section{Adrienne Bettini}

Honoraria: MSD Oncology

Consulting or Advisory Role: MSD Oncology, AstraZeneca

Travel, Accommodations, Expenses: Janssen Oncology

Martin Früh

Consulting or Advisory Role: BMS, AstraZeneca, MSD, Takeda, Roche, Lilly Speakers' Bureau: Pfizer

Research Funding: BMS, AstraZeneca

Markus Joerger

Consulting or Advisory Role: Novartis, AstraZeneca, Basilea Pharmaceutical, Bayer, Bristol Myers Squibb, Debiopharm Group, Merck, Roche, Sanofi Research Funding: AstraZeneca, Basilea Pharmaceutical, Bayer, Bristol Myers Squibb, Daiichi Sankyo, Immunophotonics, InnoMedica, Janssen Oncology, Lilly, Merck, Novartis, Pfizer, PharmaMar, Roche, Sanofi, Takeda

\section{Laetitia A. Mauti}

Consulting or Advisory Role: Takeda, Roche, AstraZeneca, Bristol Myers Squibb, Merck Sharp \& Dohme, Pfizer

Travel, Accommodations, Expenses: Takeda, Bristol Myers Squibb, Merck, Roche, AstraZeneca

\section{Christian Britschgi}

Consulting or Advisory Role: AstraZeneca, Pfizer, Roche, Takeda, Janssen-

Cilag, Boehringer Ingelheim

Travel, Accommodations, Expenses: AstraZeneca, Takeda

Walter Weder

Consulting or Advisory Role: AstraZeneca, Covidien/Medtronic

Speakers' Bureau: AstraZeneca, Covidien/Medtronic

Travel, Accommodations, Expenses: AstraZeneca, Covidien/Medtronic

Solange Peters

Honoraria: Roche, Bristol Myers Squibb, Novartis, Pfizer, MSD, AstraZeneca, Takeda, Illumina, Medscape

Consulting or Advisory Role: Roche/Genentech, Novartis, Bristol Myers Squibb, Pfizer, MSD, Amgen, AstraZeneca, Janssen, Regeneron, Merck Serono,
Boehringer Ingelheim, Takeda, Lilly, AbbVie, Bayer, Biocartis, Debiopharm Group, Illumina, PharmaMar, Sanofi, Seattle Genetics, Blueprint Medicines, Daiichi Sankyo, Incyte, Bioinvent, Clovis Oncology, Vaccibody, Phosplatin Therapeutics

Research Funding: Roche, BMS, MSD, Amgen, Lilly, AstraZeneca, Pfizer, Illumina, Merck Serono, Novartis, Biodesix, Boehringer Ingelheim, lovance Biotherapeutics, Phosplatin Therapeutics

Travel, Accommodations, Expenses: Roche, Bristol Myers Squibb, MSD, Sanofi, Incyte

Uncompensated Relationships: Journal of Thoracic Oncology, ESMO,

European Thoracic Oncology Platform (ETOP), Annals of Oncology

Michael Mark

Consulting or Advisory Role: Roche, AstraZeneca, Takeda, BMS, MSD Oncology Travel, Accommodations, Expenses: Pfizer, Roche, Takeda

\section{Richard Cathomas}

Honoraria: Janssen-Cilag, Astellas Pharma, BMS, Debiopharm Group

Consulting or Advisory Role: Astellas Pharma, Bristol Myers Squibb, Pfizer, Roche, MSD Oncology, Janssen-Cilag, Bayer, Sanofi, Ipsen

Travel, Accommodations, Expenses: AstraZeneca

Adrian F. Ochsenbein

Consulting or Advisory Role: Tolremo

Research Funding: argenx

Patents, Royalties, Other Intellectual Property: Patent on the anti-CD70 antibody cusatuzumab

Wolf-Dieter Janthur

Consulting or Advisory Role: Roche, Takeda, MSD, Novartis

Nicolas Mach

Stock and Other Ownership Interests: MaxVAX SA

Research Funding: MaxiVax

Patents, Royalties, Other Intellectual Property: I am an inventor on patent owned by MaxiVAX SA and on patent co-owned by Geneva University Hospital and MaxiVAX SA

Uncompensated Relationships: MaxiVax

Patrizia Froesch

Consulting or Advisory Role: Pfizer, Takeda, Roche, Boehringer Ingelheim, AstraZeneca, Novartis, Bayer

\section{Pierre Bohanes}

Honoraria: MSD, Bayer

Travel, Accommodations, Expenses: Janssen

Corinne Rusterholz

Employment: Roche

Miklos Pless

Honoraria: Janssen-Cilag

Consulting or Advisory Role: AbbVie, AstraZeneca, Boehringer Ingelheim, Bristol Myers Squibb, Eisai Europe, MSD Oncology, Novartis, Pfizer/EMD

Serono, Roche, Takeda, Merck Serono

Expert Testimony: Takeda

Travel, Accommodations, Expenses: Vifor Pharma, Bristol Myers Squibb, Boehringer Ingelheim, AstraZeneca

No other potential conflicts of interest were reported. 


\section{APPENDIX}

TABLE A1. PD-L1 Expression Status on Tumor Cells

PD-L1 Expression

Patients $(n=67)$, No. (\%)

\begin{tabular}{ll}
\hline$\geq 50 \%$ & $12(18)$ \\
\hline$\geq 25 \%$ & $13(19)$ \\
\hline$\geq 1 \%$ & $32(48)$ \\
\hline$<1 \%$ & $17(25)$ \\
\hline Unknown & $18(27)$
\end{tabular}

Abbreviation: PD-L1, programmed cell death-ligand 1.

TABLE A2. Radiographic Response

Response

\begin{tabular}{ll}
\hline $\mathrm{CR}$ \\
\hline $\mathrm{PR}$ \\
\hline Objective response \\
\hline $\mathrm{SD}$ \\
\hline $\mathrm{PD}$ \\
\hline $\mathrm{NE}$
\end{tabular}

After Chemotherapy ( $n=67)$, No. (\%)

2 (3)

$27(40)$

29 (43) $(95 \% \mathrm{Cl}, 31$ to 56$)$

$30(45)$

$3(5)$

2 (2)

$3(5)^{\mathrm{a}}$
After Durvalumab ( $n=62$ ), №. (\%)

4 (7)

32 (52)

36 (58) (95\% Cl, 45 to 71$)$

$16(26)$

$4(7)$

$4(7)$

$2(3)^{\mathrm{b}}$

Abbreviations: $\mathrm{CR}$, complete remission; $\mathrm{NE}$, not evaluable; $\mathrm{PD}$, progressive disease; $\mathrm{PR}$, partial remission; $\mathrm{SD}$, stable disease.

aTumor assessment not done $(n=1)$, withdrawn by physician $(n=1)$, and patient refusal $(n=1)$.

${ }^{\mathrm{b}}$ Tumor assessment not done $(\mathrm{n}=2)$.

TABLE A3. Treatment-Related AEs During Neoadjuvant Chemotherapy $(n=67)$

AE

Grades 1 and 2

Grade 3

Grade 4

Hematologic

Anemia

$3(5)$

2 (3)

Febrile neutropenia

$3(5)$

Neutropenia

2 (3)

$6(9)$

$6(9)$

Thrombocytopenia

5 (8)

$1(2)$

Nonhematologic

\begin{tabular}{lcc}
\hline Abdominal pain & $10(15)$ & $2(3)$ \\
\hline Acute kidney injury & $10(15)$ & $2(3)$ \\
\hline Alopecia & $36(54)$ & $1(2)$ \\
\hline Anaphylaxis & $19(28)$ & $5(8)$ \\
\hline Anorexia & & $1(2)$ \\
\hline Atrial fibrillation & & $1(2)$ \\
\hline Catheter-related infection & $1(2)$ \\
\hline Colitis & $16(24)$ & $4(6)$ \\
\hline Constipation & $1(2)$ & $9(13)$ \\
\hline Dehydration & $27(40)$ & \\
\hline Diarrhea & $36(54)$ & \\
\hline Dysgeusia & & \\
\hline
\end{tabular}

(continued on following page) 
TABLE A3. Treatment-Related AEs During Neoadjuvant Chemotherapy $(n=67)$ (continued)

Grades 1 and 2

Grade 3

Grade 4

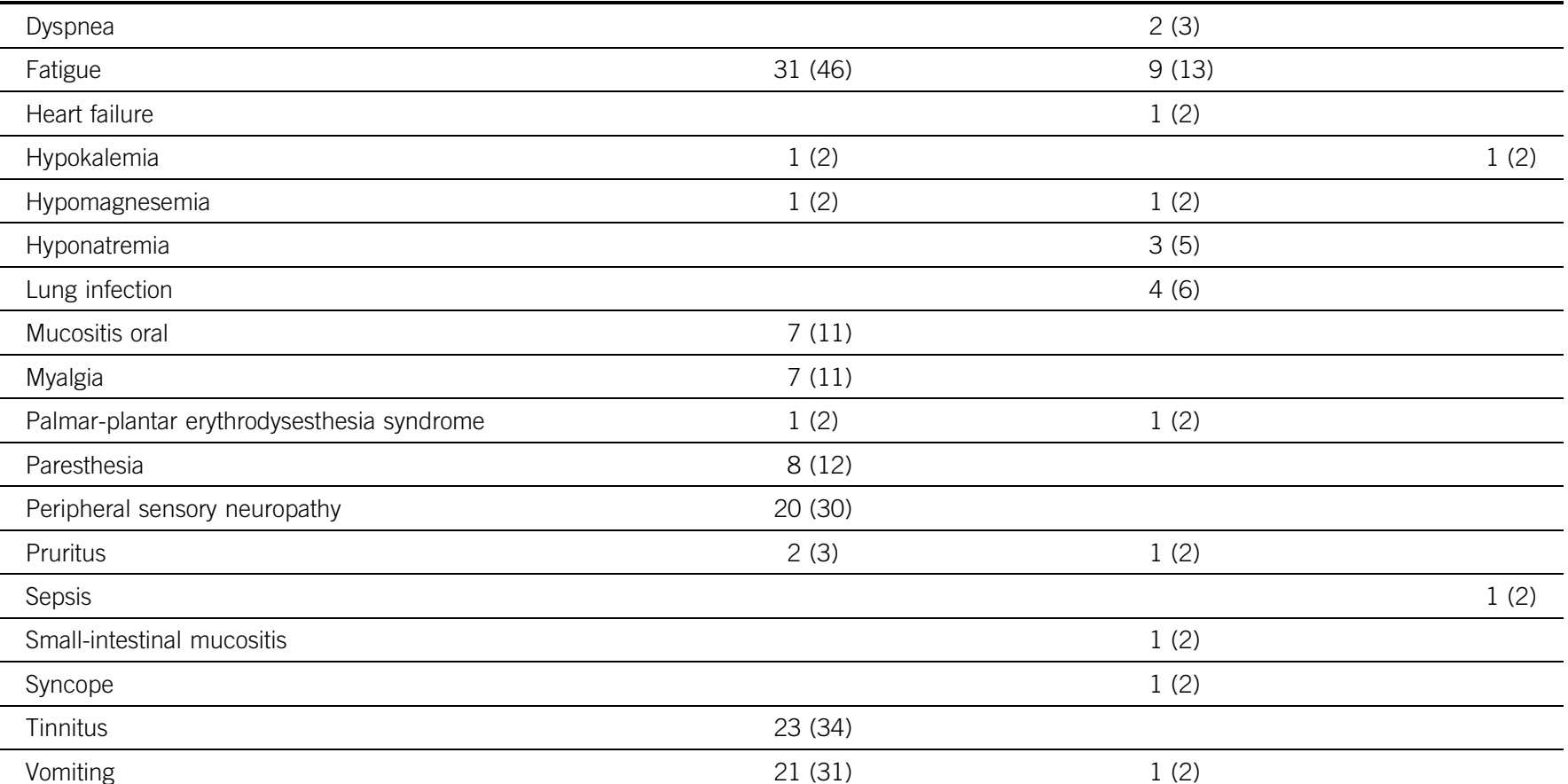

NOTE. Data are presented as No. (\%). Shown are the treatment-related AEs of any grade that occurred in more than $10 \%$ of patients or any treatmentrelated AEs of grade 3 or higher. No grade 5 treatment-related AEs were observed.

Abbreviation: $A E$, adverse event. 